\title{
Solving Nonlinear Equations Using the Laplace Decomposition Method: An Application to Projectile Motion with Resistive Term
}

Emad K. Jaradat*

Physics Department-Mutah University-Al-Karak-Jordan; ejaradat@mutah.edu.jo

\begin{abstract}
Objectives: An approximate solution of the problem of two-dimensional projectile motion with linear and quadratic air resistance obtained using the Laplace decomposition method LDM. Methods/Statistical Analysis: In this study, we examined the Laplace Decomposition Method for obtaining a solution of the trajectory problem of a body in linear and quadratic resistant medium. Findings: The solutions are obtained by using developed technique that combine Laplace Transform and the Adomian Decomposition Method ADM. The all obtained solutions are plotted to compare the effect of the nonlinear term on the basic parameters like range and maximum high and other parameters. Application/Improvements: The Laplace Decomposition Method improves the results to achieve more precision results compared to the previous related works.
\end{abstract}

Keywords: Application, Laplace Decomposition Method, Nonlinear Equations, Projectile Motion, Resistive Term

\section{Introduction}

Nonlinear phenomena equations emerge in a wide assortment of unadulterated and connected science: oscillations of mechanical structure, the motion of particles in accelerators, planetary motion $\frac{1-3}{}$. So many mathematical methods have been developed for solving these problems like: inverse method, Adomian method, perturbation method, Backland method, variational method, and Decomposition Method ${ }^{4-10}$. $\operatorname{In}^{11}$ used Laplace Decomposition Method for finding an approximate solution of a class nonlinear ordinary differential equation. In $\frac{12}{}$ exploited this method to solve FalknerSkan equation. $\operatorname{In}^{\frac{13}{3}}$ applied the same technique to obtain an approximate solution of the Duffing equation.

The shot movement through a resistive medium is one of the easiest issues whose analogs are far reaching in material science. The issue of shot movement through a resistive medium had been considered widely in various aspect. Timmerman investigated this problem of a vertically thrown ball, with a drag drive, which is either direct or quadratic in the speed. Warburton etd is played a detailed discussion of a shot movement with quadratic air resistance in various approximations $\frac{14-18}{}$. In this work, we consider the Laplace strategy for finding a surmised answer for both speed and position of the shot movement in both flat and vertical case.

\section{Laplace Decomposition Method}

The Adomian strategy, presented by George A. has enhanced to be an intense strategy to locate the surmised answers for a wide class of partial differential equations, this procedure is known as the Laplace Decomposition MethodLDM 2,9 .

To outline the essential thought of this procedure, we consider the general type of the second request nonlinear differential equation with introductory conditions given by

$$
\frac{d^{2} f(x)}{d x^{2}}+b_{1}(x) \frac{d f(x)}{d x}+b_{2}(x) f(x)=g(y)
$$




$$
f(0)=\alpha \frac{d f(0)}{d x}=\beta
$$

effect Laplace transform on both sides of equation (1):

$$
\begin{aligned}
L[f]= & \frac{\alpha}{s}+\frac{\beta}{s^{2}}+\frac{1}{s^{2}} L[g(y)] \\
& -\frac{1}{s^{2}} L\left[b_{1}(x) \frac{d f(x)}{d x}+b_{2}(x) f\right]
\end{aligned}
$$

The Laplace decomposition method gives a series solution in the form

$$
f(x)=\sum_{n=0}^{\infty} f_{n}(x)
$$

the non-linear term can be decomposed as

$$
g(y)=N f=\sum_{n=0}^{\infty} H_{n}
$$

where, He's polynomials $H_{n}$ are given by

$$
H_{n}=\frac{1}{n !} \frac{d^{n}}{d p^{n}}\left[N\left(\sum_{n=0}^{\infty} p^{i}\left(f_{i}\right)\right)\right]_{p=0}, n=0,1,2, \ldots
$$

Substituting equation (5) in equation (2), we obtained

$$
\begin{aligned}
& L\left[\sum_{n=0}^{\infty} f_{n}\right]=\frac{a}{s}+\frac{\beta}{s^{2}}+\frac{1}{s^{2}} L\left[\sum_{n=0}^{\infty} H_{n}\right] \\
& -\frac{1}{s^{2}} L\left[b_{1}(x) \sum_{n=0}^{\infty} f_{n}+b_{2}(x) \sum_{n=0}^{\infty} f_{n}\right] \\
& L\left[f_{0}\right]=\frac{a}{s}+\frac{\beta}{s^{2}} \\
& L\left[f_{1}\right]=\frac{1}{s^{2}} L\left[H_{\circ}\right]-\frac{1}{s^{2}} L\left[\left(b_{1}(x) f_{0}\right)+b_{2}(x) f_{0}\right] \\
& L\left[f_{2}\right]=\frac{1}{s^{2}} L\left[H_{1}\right]-\frac{1}{s^{2}} L\left[\left(b_{1}(x) f_{1}\right)+b_{2}(x) f_{1}\right]
\end{aligned}
$$

In general, the main recurrencerelation given as

$$
\begin{aligned}
L\left[f_{n+1}\right]= & \frac{1}{s^{2}} L\left[H_{n}\right] \\
& -\frac{1}{s^{2}} L\left[\left(b_{1}(x) \dot{f_{n}}\right)+b_{2}(x) f_{n}\right], n \geq 0
\end{aligned}
$$

Taking the inverse Laplace transform for the two sides of equation (7), one obtains $f_{0}=G(x)$, where $G(x)$ represents the term observed from the source term and from initial condition. and

$$
\begin{aligned}
f_{n+1}(x)= & L^{-1}\left[\frac{1}{s^{2}} L\left[H_{n}\right]\right. \\
& \left.-\frac{1}{s^{2}} L\left[\left(b_{1}(x) \dot{f}_{n}\right)+b_{2}(x) f_{n}\right]\right]
\end{aligned}
$$

\section{The Adomian Polynomials for Quadratic Polynomials}

$$
F(u)=u^{2}
$$

Use $u=\sum_{n=0}^{\infty} u_{n}$

Substituting $u$ into $F(u)=u^{2}$ gives

$$
F(u)=\left(u_{\circ}+u_{1}+u_{2}+u_{3}+\ldots\right)^{2}
$$

Expanding the expansion in right-hand side gives

$$
F(u)=u_{\circ}^{2}+2 u_{\circ} u_{1}+2 u_{\circ} u_{2}+u_{1}^{2}+2 u_{\circ} u_{3}+2 u_{1} u_{2}+\ldots
$$

The expansion in the above can be reformulated by gathering all terms so that the subscripts of the parts of $u_{n}$ is the same. Rearrange $F(u)$ as

$$
\begin{aligned}
F(u) & =\left(u_{\circ}^{2}\right) \\
& +\left(2 u_{\circ} u_{1}\right)+\left(2 u_{\circ} u_{2}+u_{1}^{2}\right)+\left(2 u_{\circ} u_{3}+2 u_{1} u_{2}\right) \\
& +\left(2 u_{\circ} u_{4}+2 u_{1} u_{3}+u_{2}^{2}\right) \\
& +\left(2 u_{\circ} u_{5}+2 u_{1} u_{4}+2 u_{2} u_{3}\right)+\left(2 u_{\circ} u_{6}+2 u_{1} u_{5}+2 u_{2} u_{4}+u_{3}^{2}\right) \\
& +\left(2 u_{\circ} u_{7}+2 u_{1} u_{6}+2 u_{2} u_{5}+2 u_{3} u_{4}\right) \\
& +\left(2 u_{\circ} u_{8}+2 u_{1} u_{7}+2 u_{2} u_{6}+2 u_{3} u_{5}+u_{4}^{2}\right) \\
& +\left(2 u_{0} u_{9}+2 u_{1} u_{8}+2 u_{2} u_{7}+2 u_{3} u_{6}+2 u_{4} u_{5}\right) \\
& +\left(2 u_{0} u_{10}+2 u_{1} u_{9}+2 u_{2} u_{8}+2 u_{3} u_{7}+2 u_{4} u_{6}+u_{5}^{2}\right)+\ldots
\end{aligned}
$$

The Adomian polynomials for $F(u)=u^{2}$ can be determined as follows.

$$
\begin{aligned}
& A_{\circ}=u_{\circ}^{2} \\
& A_{1}=2 u_{\circ} u_{1} \\
& A_{2}=2 u_{\circ} u_{2}+u_{1}^{2} \\
& A_{3}=2 u_{0} u_{3}+2 u_{1} u_{2} \\
& A_{4}=2 u_{0} u_{4}+2 u_{1} u_{3}+u_{2}^{2} \\
& A_{5}=2 u_{\circ} u_{5}+2 u_{1} u_{4}+2 u_{2} u_{3}
\end{aligned}
$$




$$
\begin{aligned}
& A_{6}=2 u_{0} u_{6}+2 u_{1} u_{5}+2 u_{2} u_{4}+u_{3}^{2} \\
& A_{7}=2 u_{0} u_{7}+2 u_{1} u_{6}+2 u_{2} u_{5}+2 u_{3} u_{4} \\
& A_{8}=2 u_{0} u_{8}+2 u_{1} u_{7}+2 u_{2} u_{6}+2 u_{3} u_{5}+u_{4}^{2} \\
& A_{9}=2 u_{0} u_{9}+2 u_{1} u_{8}+2 u_{2} u_{7}+2 u_{3} u_{6}+2 u_{4} u_{5} \\
& A_{10}=2 u_{0} u_{10}+2 u_{1} u_{9}+2 u_{2} u_{8}+2 u_{3} u_{7}+2 u_{4} u_{6}+u_{5}^{2}
\end{aligned}
$$

\section{Projectile Motion with Linear and Quadratic Resistance Medium}

One of the most probable methods to discuss the trajectory motion is to analyze this motion in each direction separately. In other words, we will use one set of equations to describe the horizontal motion and another set of equations to describe the vertical one. In motion like this, the only acceleration will be in $\mathrm{z}$-direction and therefore, the horizontal velocities components will be constants.

We will begin when the shot movement is constrained by an air opposition, which specifically relies upon the speed, at that point the Newton's second law for the vertical movement is:

$$
m \dot{v}=-m g-k v, v(0)=u_{0}
$$

Apply the Laplace transform to both sides for last equation and use the initial conditions, we obtained

$$
v(s)=\frac{u_{0}}{s}-\frac{g}{s^{2}}-\frac{k}{m s} \mathcal{L}\{v\}
$$

Applying Adomian polynomials

$$
v(s)=\sum_{n=0}^{\infty} v_{n}(s) \mathbb{E}
$$

Use this definition in equation (10), we found

$$
\sum_{n=0}^{\infty} v_{n}(s)=\frac{u_{0}}{s}-\frac{g}{s^{2}}-\frac{k}{m s} \mathcal{L}\{v\}
$$

Define $v=\sum_{n=0}^{\infty} A_{n}(u)$

$$
\sum_{n=0}^{\infty} v_{n}(s)=\frac{u_{0}}{s}-\frac{g}{s^{2}}-\frac{k}{m s} \mathcal{L}\left\{\sum_{n=0}^{\infty} A_{n}(u)\right\}
$$

Now, we will find the Adomian polynomials $\mathrm{A}_{\mathrm{n}}$.

$$
A_{0}=v_{0}, A_{1}=v_{1}, A_{2}=v_{2}, A_{3}=v_{3} \text { and so on } \ldots
$$

If we match both sides of equation (13), we obtained

$$
\begin{gathered}
v_{0}(t)=\mathcal{L}^{-1}\left\{\frac{u_{0}}{s}-\frac{g}{s^{2}}\right\} \Rightarrow v_{0}(t)=u_{0}-g t \\
\mathcal{L}\left\{v_{1}(t)\right\}=\frac{-k}{m s} \mathcal{L}\left\{A_{0}\right\} \Rightarrow v_{1}(t)=\frac{-k}{m}\left(u_{0} t-\frac{1}{2} g t^{2}\right) \\
\mathcal{L}\left\{v_{2}(t)\right\}=\frac{-k}{m s} \mathcal{L}\left\{A_{1}\right\} \Rightarrow v_{2}(t)=\frac{k^{2}}{m^{2}}\left(\frac{u_{0} t^{2}}{2 !}-\frac{g t^{3}}{3 !}\right)
\end{gathered}
$$

also, we can find the rest terms to get general solution as

$$
v_{n}(t)=\frac{(-1)^{n} k^{n}}{m^{n}}\left(\frac{u_{0} t^{n}}{n !}-\frac{g t^{n+1}}{(n+1) !}\right)
$$

To obtain an approximate solution we take the summation for all terms, this yield

$$
v(t)=\sum_{n=0}^{\infty} \frac{(-1)^{n} k^{n}}{m^{n}}\left(c_{1} u_{0} t^{n}-c_{2} g t^{n+1}\right)
$$

where, $c_{1}$ and $c_{2}$ are constants that appear as a result from applying Laplace transform.

The horizontal motion will have same steps except that $g=0$, then the solution for the horizontal motion will be

$$
v(t)=\sum_{n=0}^{\infty} \frac{(-1)^{n} k^{n}}{m^{n}}\left(c_{1} u_{0} t^{n}\right)
$$

Let the projectile is forced by an air resistance which depends on the quadratic resistance medium, then the Newton's second law is:

$$
\dot{v}=-g-\frac{k}{m} v^{2} ; v(0)=u_{0}
$$

In the wake of applying Laplace transform on the two sides and taking the opposite Laplace transform, we acquire

$$
v(s)=\frac{u_{0}}{s}-\frac{g}{s^{2}}-\frac{k}{m s} \mathcal{L}\left\{v^{2}\right\}
$$

Use equation (11) and the definition of $v^{2}=\sum_{n=0}^{\infty} A_{n}(u)$ we obtain,

$$
\sum_{n=0}^{\infty} v_{n}(s)=\frac{u_{0}}{s}-\frac{g}{s^{2}}-\frac{k}{m s} \mathcal{L}\left\{\sum_{n=0}^{\infty} A_{n}(u)\right\}
$$

The related Adomian polynomials are 


$$
\begin{aligned}
& A_{0}=v_{0}^{2}, A_{1}=2 v_{0} v_{1}, A_{2}=v_{1}^{2}+2 v_{0} v_{2}, \\
& A_{3}=2 v_{1} v_{2}+2 v_{0} v_{3} \text { and so on.... }
\end{aligned}
$$

Then,

$$
\begin{gathered}
v_{0}(t)=\mathcal{L}^{-1}\left\{\frac{u_{0}}{s}-\frac{g}{s^{2}}\right\} \Rightarrow v_{0}(t)=u_{0}-g t \\
\mathcal{L}\left\{v_{1}(t)\right\}=\frac{-k}{m s} \mathcal{L}\left\{v_{0}{ }^{2}\right\} \Rightarrow v_{1}(t) \\
=\frac{-k}{m}\left(u_{0}{ }^{2} t-g u_{0} t^{2}+\frac{g^{2}}{3} t^{3}\right) \\
\mathcal{L}\left\{v_{2}(t)\right\}=\frac{-k}{m s} \mathcal{L}\left\{2 v_{0} v_{1}\right\} \Rightarrow v_{2}(t) \\
=\frac{k^{2}}{m^{2}}\left(u_{0}^{3} t^{2}-\frac{8 g u_{0}{ }^{3} t^{3}}{3 !}+\frac{16 u_{0} g^{2} t^{4}}{4 !}-\frac{16 g^{3} t^{5}}{5 !}\right) \\
v_{3}(t)=\frac{-k^{3}}{m^{3}}\left(u_{0}^{4} t^{3}-\frac{5}{3} u_{0}^{3} g t^{4}+\frac{17}{15} u_{0}^{2} g^{2} t^{5}\right. \\
\left.-\frac{17}{45} u_{0} g^{3} t^{6}+\frac{17}{315} g^{4} t^{7}\right) \\
v_{n}(t)=\frac{(-1)^{n} k^{n}}{m^{n}}\left(u_{0}{ }^{n+1} g^{0} t^{n}-u_{0}{ }^{n} g^{1} t^{n+1}\right. \\
\left.+u_{0}{ }^{n-1} g^{2} t^{n+2}-\ldots+u_{0}^{0} g^{n+1} t^{2 n+1}\right) \\
\frac{k^{4}}{m^{4}}\left(u_{0}^{5} t^{4}-2 g u_{0}{ }^{4} t^{5}+\frac{77}{45} u_{0}^{3} g^{2} t^{6}\right. \\
\left.-\frac{248}{315} u_{0}{ }^{2} g^{3} t^{7}+\frac{62}{315} u_{0} g^{4} t^{8}-\frac{62}{2835} g^{5} t^{9}\right)
\end{gathered}
$$

Then theseries solution for the vertical shot movement takes the form

$$
\begin{aligned}
& v(t)=\sum_{n=0}^{\infty} \frac{(-1)^{n} k^{n}}{m^{n}}\left(b_{1} u_{0}^{n+1} g^{0} t^{n}-b_{2} u_{0}^{n} g^{1} t^{n+1}\right. \\
& +b_{3} u_{0}^{n-1} g^{2} t^{n+2}-\ldots \\
& \left.+b_{a} u_{0}^{0} g^{n+1} t^{2 n+1}\right) \\
& \mathrm{b}_{1} \text { to } \mathrm{b}_{\alpha} \text { are constants }
\end{aligned}
$$

And the solution for horizontal one is

$$
v(t)=\sum_{n=0}^{\infty} \frac{(-1)^{n} k^{n}}{m^{n}}\left(b_{1} u_{0}^{n+1} t^{n}\right)
$$

The strong line represents the solution which is gotten by LDM and the dashed one represent the solution which is acquired by common method depending on integration with respect to time.

For comparison, we additionally plot all arrangements of the shot movement equation with quadratic air obstruction which is tackled in two strategies. Figures 1-3 show how our technique leads to the same solution that was obtained by common methods to solve nonlinear differential equations. This comparison gives an idea about effectiveness and successful of Laplace decomposition method, especially if we consider that the approximate solution will be for the summation of all terms. The small errors in the figure refers to neglecting the rest terms of the summation.

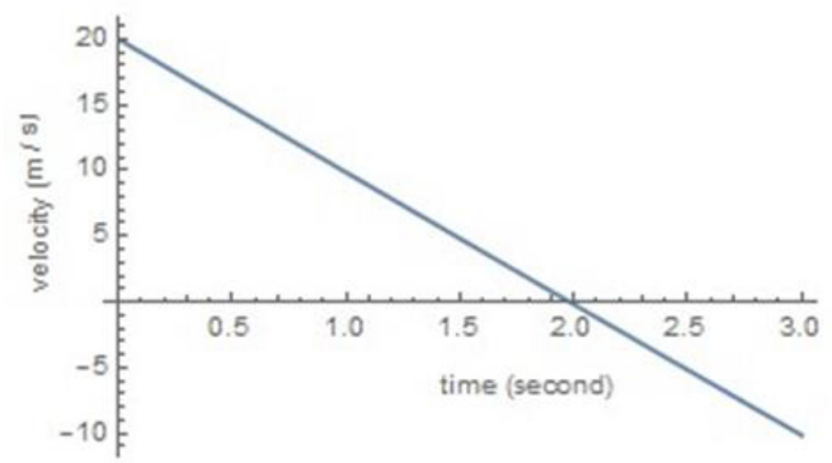

Figure 1. Velocity with linear air resistance for mass $m=20 g, k=0.2, v_{\mathrm{o}}=40 m s^{-1}$.

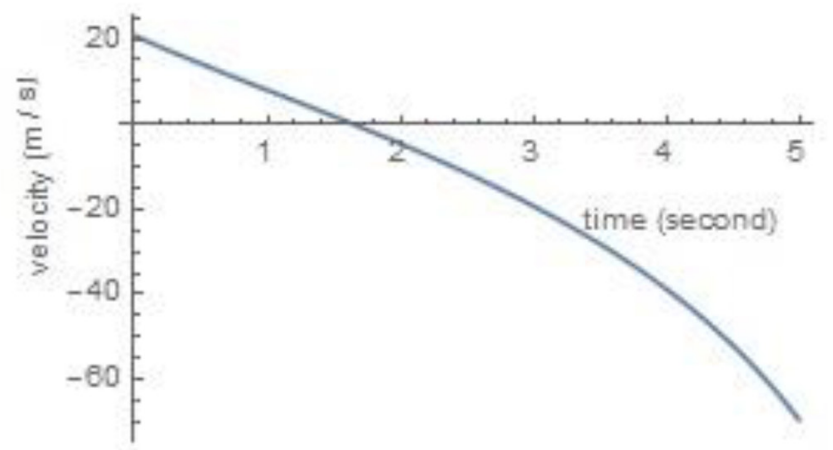

Figure 2. Velocity with quadratic air resistance for mass $m=20 g, k=0.2, v_{\circ}=40 \mathrm{~ms}^{-1}$. 


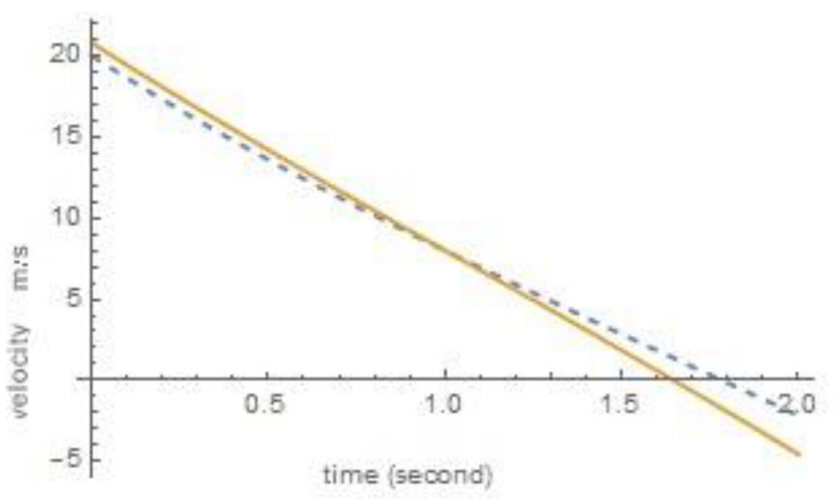

Figure 3. Velocity for projected mass, $=10 \mathrm{~g},=0.1, v_{\mathrm{o}}=20$ $\mathrm{m} / \mathrm{s}$ with quadratic air resistance. The solid line represents the solution that obtained by LDM and the dashed one represent the solution that obtained by common method depending on integration with respect to time.

\section{Conclusion}

An approximate solution of the problem of the trajectory motion with resistance obtained using the Laplace decomposition method LDM. These series solutions are expressed as simple power for each horizontal and vertical motion for both linear and quadratic air resistance. The acquired arrangements are plotted to consider the effect of the nonlinear term, and to contrast other arrangement that was gotten by different strategies. These outcomes create the impression that the LDM is a viable and effective technique to get an approximate answer for comparable nonlinear physical Phenomena.

\section{References}

1. Fowls GR, Cassidy GL. Analytical mechanics, University of Utah, 7th edition; 2004.

2. Wang M, Zhou Y. Application of a homogeneous method to exact solutions of Non-linear equations. Physics Letter A. 1996 June 17; 216(1-5)67-75.

3. George A. Non-linear stochastic operator equations. Academic Press, 1st Edition; 1986.

4. Kazuki Y. An analytic solution of projectile motion with the quadratic resistance law using the homotopy analysis method. Journal of Physics A: Mathematical and Theoretical. 2007; 40(29).

5. Ryogo H. A new form of Backlund transformations and its relation to the inverse scattering problem. Progress of Theoretical Physics. 1974; 52(5):1-15.

6. Matveev VB. Darboux Transformations and Solitons. Springer-Verlag; 1991. https://doi.org/10.1007/978-3-662 00922-2

7. Abdul-Majid W. The tanh method for compact and noncompact solutions for variants of KdVBurger and the $\mathrm{K}$ (n, n) equations. Physics D. 2006; 213(2).

8. George A. Solving frontier problems of physics: the decomposition method. Theoretical, Mathematical and Computational Physics. 2013, 60. PMid:23623364

9. George A. A review of the decomposition method, some recent results for non-linear equations. Mathematical and Computer Modelling. 1990; 13(7):17-43. https://doi. org/10.1016/0895-7177(90)90125-7

10. Sujit, H. Karande. Application of LDM to Solve Linear and Non-linear Heat Equation. International Journal of Applied Physics and Mathematics. 2012; 2(5):1-3.

11. Suheil K. Laplace decomposition algorithm applied to class of non-linear diffequs. Journal of Applied Mathematics. 2001; 1:141-55. https://doi.org/10.1155/S1110757X01000183

12. Nasser S. Numerical solution for the Falkner-Skan equation. Chaos, Solitons and Fractals. 2008; 35:733-46.

13. Elçin Y. Numerical Solution of Duffing Equation by the LDA. Applied Mathematics and Computation; 2006. p. 572-80.

14. Warburton DH, Wang J, J. Burgdörfer. Analytic approximations of projectile motion with air resistance. Scientific Research. 2010; 3:98-105. https://doi.org/10.4236/jssm. 2010.31012

15. Parker GW. Projectile motion with air resistance quadratic in the speed. American Journal of Physics.1977; 45(7). https://doi.org/10.1119/1.10812

16. Tan A, Edwards ME. The jerk vector in projectile motion. The Latin-American Journal of Physics Education. 2011; 5:344-47.

17. Alexandre V. Horizontal distance travelled by a mobile experiencing a quadratic drag force. European Journal of Physics. 2007; 28.

18. Peter T, Van der Weele. On the rise of a ball with linear or quadratic drag. American Journal of Physics. 1999; 67: 538-46. https://doi.org/10.1119/1.19320 